\title{
Science meets magic: photonic metamaterials
}

\author{
Ekmel Ozbay
}

\section{SPIE.}




\title{
Science Meets Magic: Photonic Metamaterials
}

\author{
Ekmel Ozbay
}

Nanotechnology Research Center, Department of Physics, and Department of Electrical and Electronics Engineering, Bilkent University, Bilkent, 06800 Ankara, Turkey

"ozbay@,bilkent.edu.tr

\begin{abstract}
The word "magic" is usually associated with movies, fiction, children stories, etc. but seldom with the natural sciences. Recent advances in metamaterials have changed this notion, in which we can now speak of "almost magical" properties that scientists could only dream about only a decade ago. In this article, we review some of the recent "almost magical" progress in the field of meta-materials.
\end{abstract}

Keywords: metamaterials, left-handed metamaterial, negative refractive index, superlens.

\section{INTRODUCTION}

In recent years, there has been a burgeoning interest in rapidly growing field of metamaterials due to their unprecedented properties unattainable from ordinary materials. Veselago pointed out that a ma-terial exhibiting negative values of dielectric permittivity $(\varepsilon)$ and magnetic permeability $(\mu)$ would have a negative refractive index [1]. Generally speaking, the dielectric permittivity $(\varepsilon)$ and the magnetic permeability $(\mu)$ are both positive for natural materials. In fact, it is possible to obtain negative values for $\varepsilon$ and $\mu$ by utilizing proper designs of metamaterials. Left-handed electromagnetism and negative refraction are achievable with artificially structured metamaterials exhibiting negative values of permittivity and permeability simultaneously at a certain frequency region. The first steps to realize these novel type of materials were taken by Smith et al., where they were able to observe a left-handed propagation band at frequencies where both dielectric permittivity and magnetic permeability of the composite metamaterial are negative [2].

\section{SUBWAVELENGTH RESOLUTION}

Pendry conceived that a negative index material could be a potential candidate for imaging beyond the diffraction limit [3]. Negative index materials can restore the amplitude of evanescent waves and therefore enable subwavelength focusing [4]. Here, we employ the LHM under investigation in imag-ing measurement. In the imaging experiments, we used monopole antennae to imitate the point source. The exposed center conductor acts as the transmitter and receiver and has a length of $4 \mathrm{~cm}(\sim \lambda / 2)$. The full width at half maximum (FWHM) of the beam is $8.2 \mathrm{~cm}$

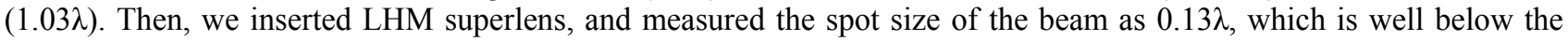
diffraction limit [5]. Since we were able to image a single point source with a subwavelength spot size, we used two point sources separated by distances smaller than a wavelength to obtain subwavelength resolution. The sources are driven by two independent signal generators and the power distribution is detected by using a microwave spectrum analyzer. The frequencies of the sources differed by $1 \mathrm{MHz}$ to ensure that the sources are entirely incoherent. The imaging experiments are performed for two different separation distances between the sources. The imaged peaks were easily resolved when the sources were separated by $\lambda / 8$. We then increased the separation of the sources to a distance of $\lambda / 5$ and the peaks were resolved better. When the sources were $\lambda / 3$ apart, we were able to resolve two peaks entirely.

Photonic and Phononic Properties of Engineered Nanostructures II,

edited by Ali Adibi, Shawn-Yu Lin, Axel Scherer, Proc. of SPIE Vol. 8269

82690L - (c) 2012 SPIE - CCC code: 0277-786X/12/\$18 - doi: 10.1117/12.916557

Proc. of SPIE Vol. 8269 82690L-1 


\section{ENHANCED TRANSMISSION}

We obtained enhanced transmission of electromagnetic waves through a single subwavelength aper-ture by making use of the resonance behavior of a split ring resonator (SRR) at microwave frequen-cies. By placing a single SRR at the near-field of the aperture, strongly localized electromagnetic fields were effectively coupled to the aperture with a radius that is twenty times smaller than the reso-nance wavelength $(\mathrm{r} / \lambda=0.05)$. We obtained 740 -fold transmission enhancement by exciting the elec-tric resonance of SRR [6].

We have recently extended this transmission enhancement performance to even higher values [7]. The respective design parameters of the connected SRRs (Sample A\&B) as depicted in Figure 1. The dis-tinct SRR samples are deposited on a dielectric printed circuit board (PCB). We drilled an opening on a large metal plate. The dimensions of the metal plate were intentionally picked to be large in order to minimize the diffraction effects at the edges. The opening on our metal screen constituted the sub-wavelength aperture. The connected SRRs are inserted inside the aperture. We tried to manually align the samples to the midpoint of the aperture while leaving equal portions on both half planes. Trans-mission measurements were performed with conventional horn antennas operating around the frequency band of our interest. Transmission peaks that appeared in the simulation results suggested transmission enhancement. We also verified the transmission enhancement phenomenon experiment-ally. Our experiments indicated a transmission improvement factor above 70000 through a subwave-length aperture with a width of $\lambda / 31$ and a height of $\lambda / 12$ in terms of the operational wavelength. We tried to emphasize the role of the connecting bars during this process. We numerically showed that the connecting bars linked the otherwise isolated SRRs to each other and guided the incoming wave through the subwavelength hole. The highly localized fields around the aperture, owing to the mag-netic resonance of the SRRs, efficiently coupled the input wave to the exit side. This approach brings in the opportunity of attaining even higher transmission improvement factors by minimizing the de-pendence on the aperture geometry.

(a)

(b)
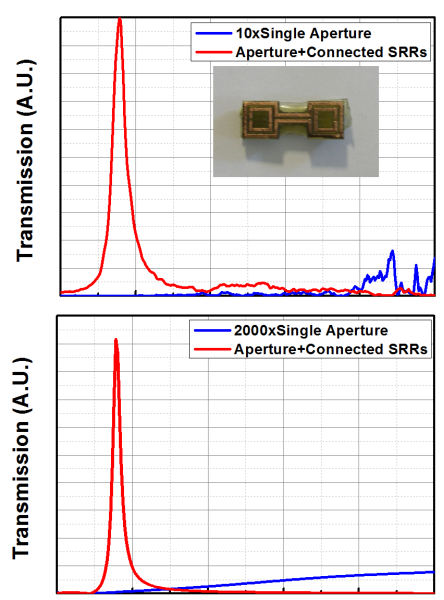

(c)

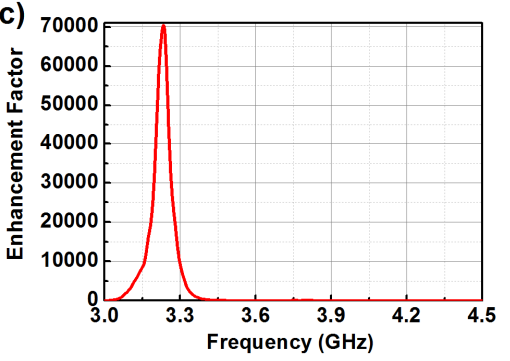

(d)

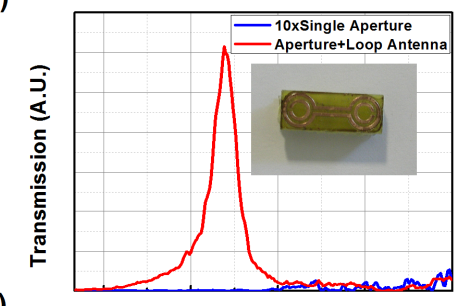

(e)
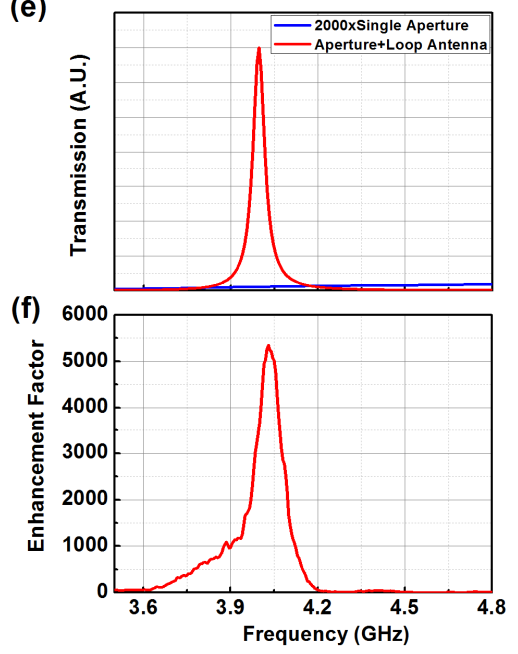

Fig. 1: (a) Measured, (b) simulated transmission results for Sample A (solid red lines) and the aperture (solid blue lines). (c) Experimentally validated enhancement factor for Sample A. (d) Measured, (e) simulated trans-mission results for Sample B (solid red lines) and the aperture (solid blue lines). (f) Experimentally validated enhancement factor for Sample B. 


\section{COMPLEMENTARY CHIRAL METAMATERIALS}

Recently, chiral metamaterials (CMMs) have attracted much attention due to their exotic properties, e.g. giant optical activity, circular dichroism, and negative refraction [9]. A CMM lacks any mirror symmetry so that the crosscoupling between the electric and magnetic fields exists at the resonance. We experimentally and numerically report a complementary bilayer cross-wire chiral metamaterial. It exhibits giant optical activity and a small circular dichroism. Figure 2 shows the retrieved effective parameters of the chiral metamaterials based on the simulation (left) and experimental (right) data. The retrieval results reveal that a negative refractive index is realized for right circularly polarized waves due to the strong chirality. Our numerical results show that the mechanism of the chiral behav-ior at the resonance of lower frequency can be interpreted as the coupling effects between two sets of mutually twisted virtual magnetic dipoles, while the resonance of higher frequency shows complicated nonlocal features. This demonstrates both experimentally and numerically, the chiral properties of a CCMM. Like its counterpart (the cross-wire CMM), the CCMM also shows giant optical activity, and due to the strong chirality, the frequency bands of negative index are obtained for RCP waves. Our numerical results show that the mechanism of the chiral behavior at the resonance of lower frequency can be interpreted as the coupling effects between two sets of mutually twisted virtual magnetic di-poles, but the resonance of higher frequency shows complicated nonlocal features.

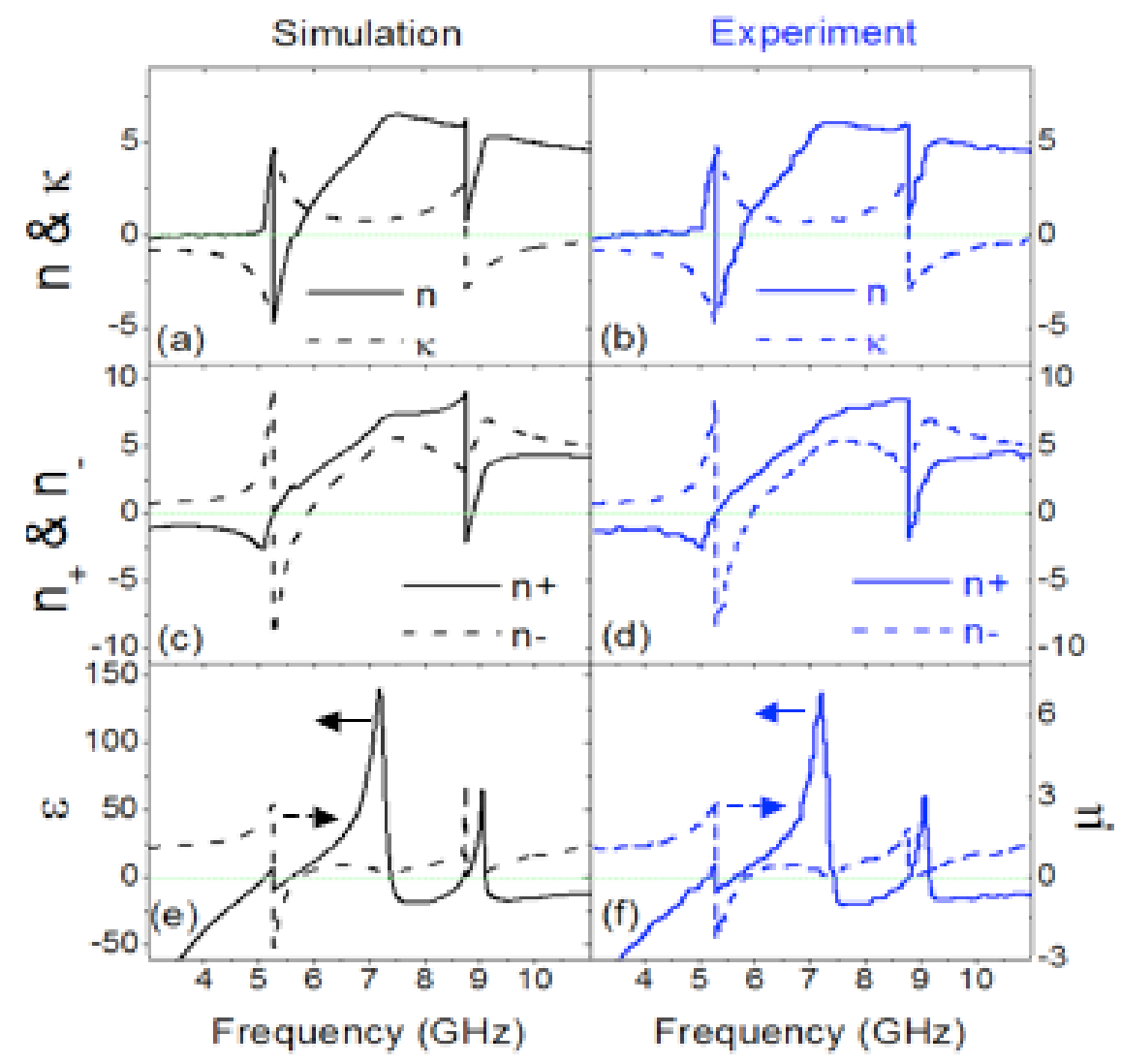

Fig. 2. The retrieved effective parameters of the chiral metamaterials based on the simulation (left) and experimental (right) data. (a) and (b) show the real parts of the refractive index $n$ and chirality $\kappa$. (c) and (d) show the real parts of the refractive indices for RCP and LCP waves. (e) and (f) show the real parts of the permittivity $\varepsilon$ and permeability $\mu$. 


\section{ASYMMETRIC TRANSMISSION USING CHIRAL METAMATERAILS}

Asymmetric transmission of electromagnetic (EM) waves is an electromagetic phenomenon that has been studied for different structures in several studies. [10] An electrically thin chiral metamaterial structure composed of four U-shaped split ring resonator pairs is utilized in order to realize polarization rotation that is dependent on the polarization of the incident wave at $6.2 \mathrm{GHz}$. The structure is optimized such that a plane wave that is linearly polarized at an arbitrary angle is an eigenwave of the system at this frequency. The analytical relation between the incident polarization and the polarization rotation is derived using transmission matrices. Furthermore, the proposed structure exhibits an asymmetric transmission of linearly polarized waves at $6.2 \mathrm{GHz}$. Figure 3 shows that at several angles, $-\mathrm{z}$ and $+\mathrm{z}$ propagating waves are transmitted symmetrically, with the same polarization. Plane waves traveling in opposite but perpendicular directions to the material plane are rotated by different angles. On the other hand, four incident polarization angles have been found for the same structure, at which the transmission is symmetric. The experiment results are in good agreement with the numerical results. [11]
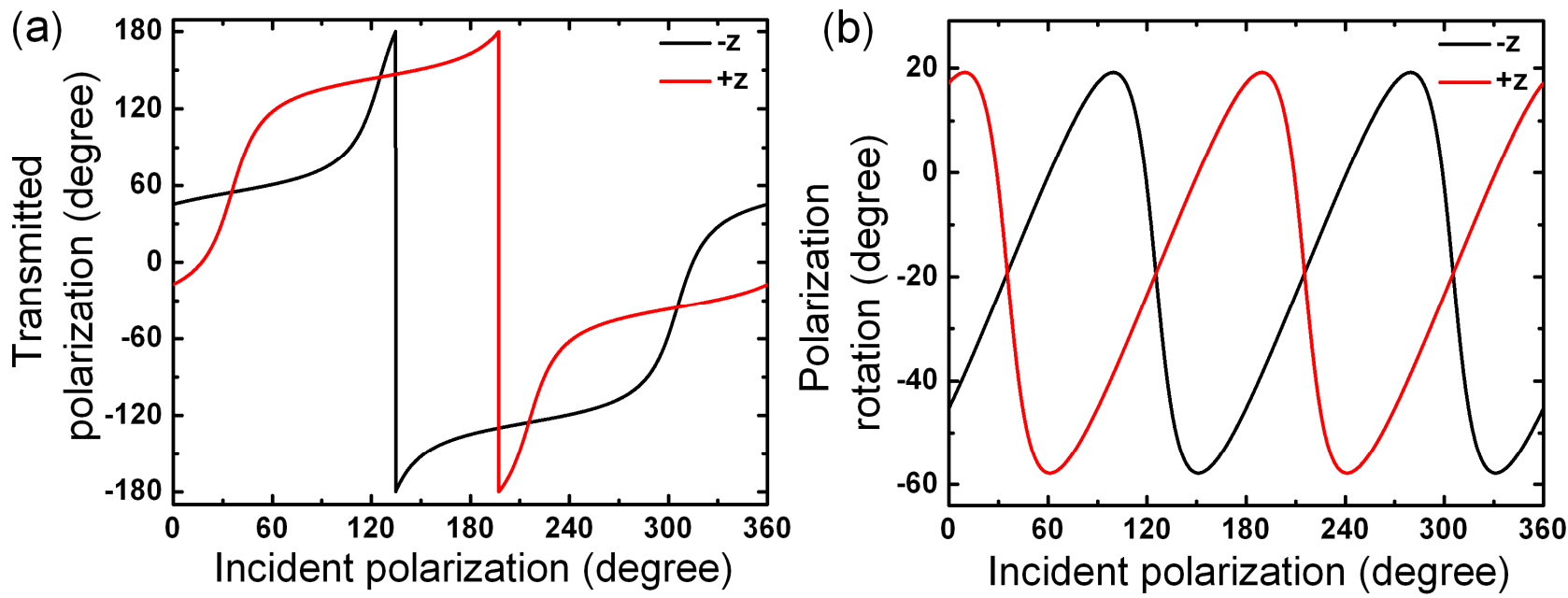

Fig. 3. (a) Polarization angles of the transmitted linearly polarized waves with respect to the polarization angle of the incident wave, at $6.2 \mathrm{GHz}$, for the $-\mathrm{z}$ and $+\mathrm{z}$ propagating waves (b) Introduced polarization rotation to the $-\mathrm{z}$ and $+\mathrm{z}$ propagating waves with respect to the incident polarization angle, at $6.2 \mathrm{GHz}$.

\section{OPTICALLY THIN INFRARED ABSORBERS USING METAMATERAILS}

Artificial periodic structures, such as photonic crystals, plasmonic structures, and metamaterials, provide for the extensive control of light. Perfect absorption was demonstrated by utilizing structures with resonant metallic unit cells at various frequencies of the spectrum. [12] We designed, fabricated, and experimentally characterized thin absorbers utilizing both electrical and magnetic impedance matching at the near-infrared regime. The absorbers consist of four main layers: a metal back plate, dielectric spacer, and two artificial layers. The presence of the titanium layer is of critical importance for the broadband operation. In Fig. 4, the blue dotted line corresponds to a super-cell with gold-only SRRs. We can clearly see that the addition of a correctly tuned Ti SRR layer shifted the gold-only SRR peak to red and also broadened the bandwidth.One of the artificial layers provides electrical resonance and the other one provides magnetic resonance yielding a polarization independent broadband perfect absorption. The structure response remains similar for the wide angle of incidence due to the sub-wavelength unit cell size of the constituting artificial layers. The design is useful for applications such as thermal photovoltaics, sensors, and camouflage. [13] 


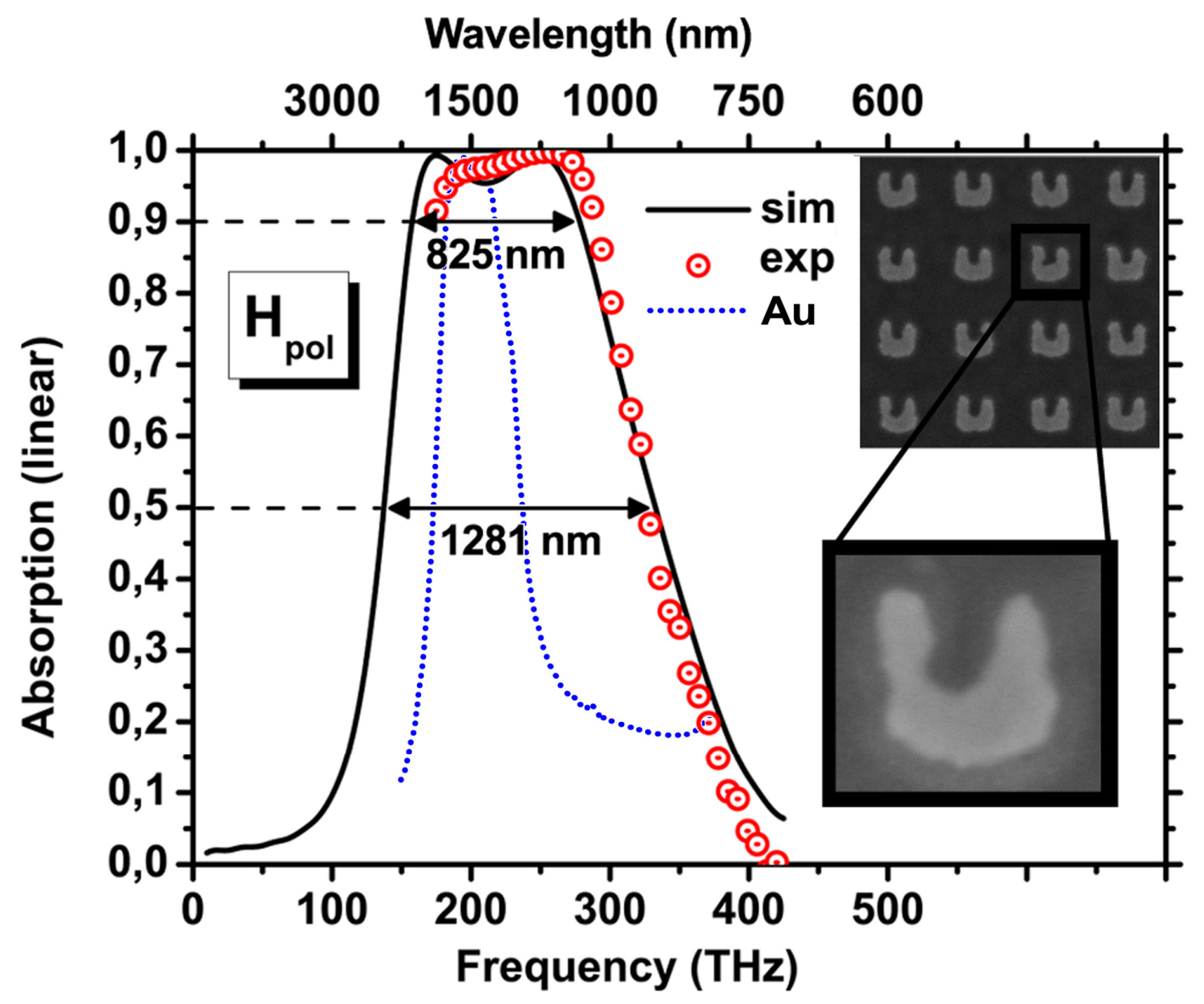

Fig. 4. Numerical and experimental data of absorbance derived from scattering parameters. The dotted line corresponds to gold-only SRR layer performance. The SEM image of a section of the printed area and an example SRR are shown on the right.

\section{SWITCHABLE METAMATERIALS AT THZ WAVELENGTHS}

Terahertz (THz) technology has become a hopeful alternative for sensing, imaging, and applications such as amplifiers, modulators, and switches. One of the greatest obstacles in this progress is the non-existence of materials that naturally respond well to $\mathrm{THz}$ radiation. One of the advantages of metamaterials for $\mathrm{THz}$ applications is their resonant electromagnetic response, which significantly enhances their interaction with $\mathrm{THz}$ radiation. We experimentally demonstrated, for the first time, an optically-implemented blue-shift tunable metamaterial in the terahertz (THz) regime. The design implies two potential resonance states, and the photo-conductive semiconductor (silicon) settled in the critical region plays the role of intermediary for switching the resonator from mode $1(0.76 \mathrm{THz})$ to mode $2(0.96 \mathrm{THz})$. Figure 5 shows the experimental measurements and theoretical simulations for the $\mathrm{THz}$ metamaterial structures under different optical excitations. The observed tuning range of the fabricated device is as high as $26 \%$ through optical control to silicon. The realization of broadband blue-shift tunable metamaterial offers opportunities for achieving switchable metamaterials with simultaneous red- and blue-shift tunability and cascade tunable devices. Our experimental approach is compatible with semiconductors technologies and can be used for other applications in the THz regime. The work also paves the way for future bi-directional (simultaneously red and blue-shift) and multi-band even cascade switchable devices, which especially benefits various important THz applications in filling the so-called THz gap". [14] 

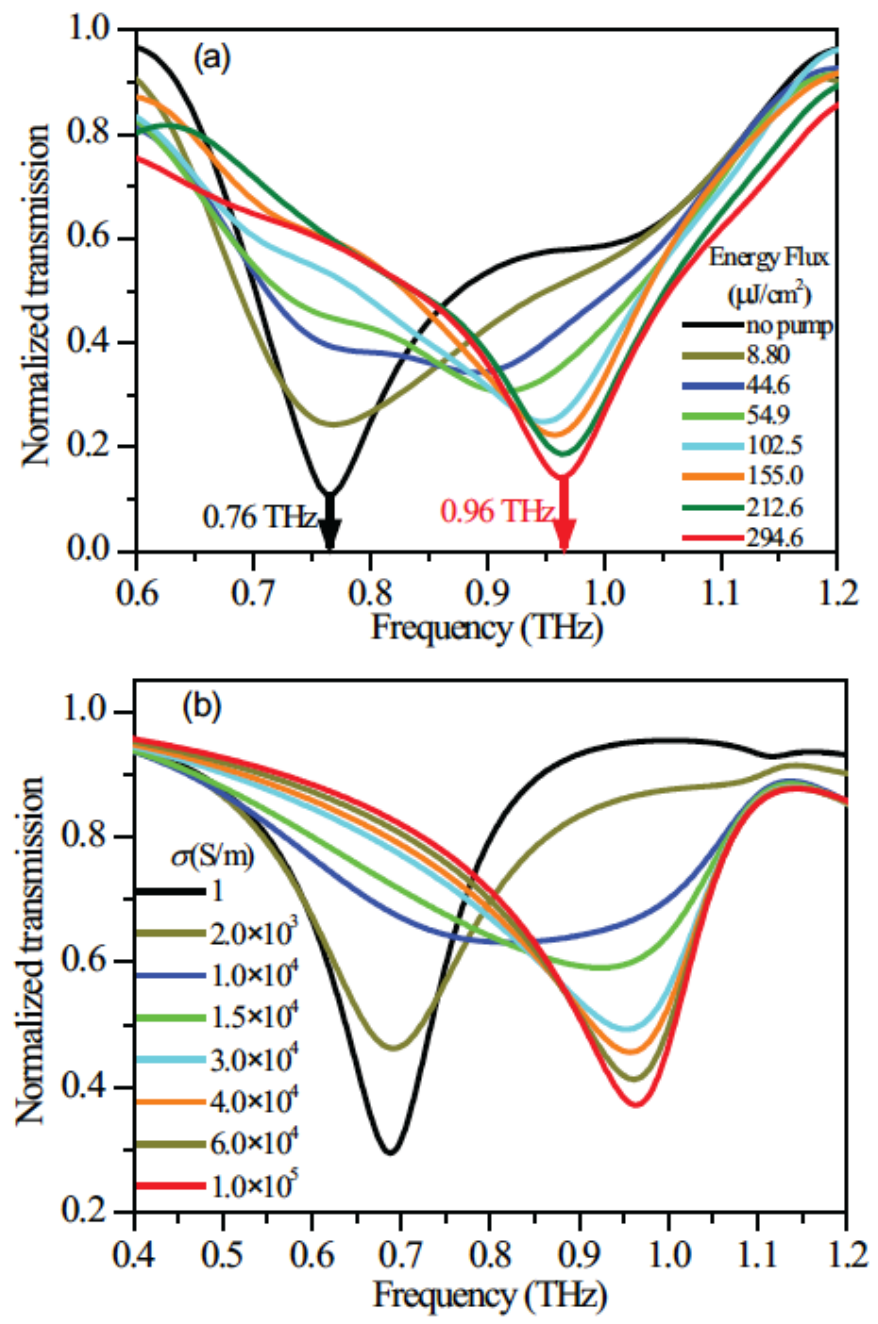

Fig. 5. Normalized transmission amplitude of the THz beam for the metamaterial device (a) Results of experimental measurements as a function of energy flux of pump beam; (b) Results of simulations as a function of conductivity of silicon.

\section{CONCLUSION}

Since the start of their very early demonstration back in 2001, metamaterials have captured the imagination of not only the scientists but also the public. After a decade of continuous metamaterial based innovations, the field is still quite very active where new "almost magical" properties of metamaterials are being discovered. This momentum is expected to continue well into the second decade of this new class of materials with new properties that we can not imagine today.

\section{ACKNOWLEDGMENT}

This work is supported by the projects DPT-HAMIT, ESF-EPIGRAT, EU-N4E, NATO-SET-181 and TUBITAK under Project Nos., 107A004, 107A012, 109E301. We also acknowledge partial support from the Turkish Academy of Sciences. 


\section{References}

[1] V. G. Veselago, "The electrodynamics of substances with simultaneously negative values of permittivity and permeability," Sov. Phys. Usp. 10, 509 (1968).

[2] D. R. Smith, W. J. Padilla, D. C. Vier, S. C. Nemat-Nasser, and S. Schultz, "Composite medium with simultaneously negative permeability and permittivity," Phys. Rev. Lett. 84, 4184-4187 (2000).

[3] J. B. Pendry, "Negative refraction makes a perfect lens," Phys. Rev. Lett. 85, 3966-3969 (2000).

[4] K. Aydin, K. Guven, C. M. Soukoulis, and E. Ozbay, "Observation of negative refraction and negative phase velocity in left-handed metamaterials," Appl. Phys. Lett. 86, 124102 (2005).

[5] K. Aydin, I. Bulu, and E. Ozbay, "Subwavelength resolution with a negative-index metamaterial superlens" Appl. Phys. Lett. 90, 254102 (2007).

[6] Koray Aydin, A. Ozgur Cakmak, Levent Sahin, Zhaofeng Li, Filiberto Bilotti, Lucio Vegni, and Ekmel Ozbay, "Split-Ring-Resonator-Coupled Enhanced Transmission through a Single Subwavelength Aperture," Physical Review Letters 102, 013904 (2009).

[7] Damla Ates, Atilla Ozgur Cakmak, Evrim Colak, Rongkuo Zhao, C. M. Soukoulis, and Ekmel Ozbay, "Transmission enhancement through deep subwavelength apertures using connected split ring resonators" Optics Express, Volume 18, 3952 (2010)

[8] S. Zhang, Y.-S. Park, J. Li, X. Lu, W. Zhang, and X. Zhang, "Negative Refractive Index in Chiral Metamaterials," Phys. Rev. Lett. 102, 023901 (2009).

[9] Z. Li, H. Caglayan, E. Colak, J. Zhou, C. M. Soukoulis, and E. Ozbay, "Coupling effect between two adjacent chiral structure layers," Opt. Express 18, 5375 (2010).

[10] C. Menzel, C. Helgert, C. Rockstuhl, E. B. Kley, A. Tünnermann, T. Pertsch, and F. Lederer, "Asymmetric transmission of linearly polarized light at optical metamaterials" Phys. Rev. Lett. 104, 253902 (2010).

[11] Mehmet Mutlu, Ahmet Emin Akosman, Andriy E. Serebryannikov, and Ekmel Ozbay, "Asymmetric chiral metamaterial circular polarizer based on four U-shaped split ring resonators," Optics Letters 36, 1653 (2011).

[12] J. Hao, J. Wang, X. Liu, W. J. Padilla, L. Zhou, M. Qiu, "High performance optical absorber based on a plasmonic metamaterial," Appl. Phys. Lett. 96, 251104 (2010).

[13] Kamil Boratay Alici, Adil Burak Turhan, Costas M. Soukoulis, and Ekmel Ozbay, "Optically thin composite resonant absorber at the near-infrared band: a polarization independent and spectrally broadband configuration," Optics Express 19, 14260 (2011).

[14] W. J. Padilla, A. J. Taylor, C. Highstrete, M. Lee, and R. D. Averitt, "Dynamical Electric and Magnetic Metamaterial Response at Terahertz Frequencies," Phys. Rev. Lett. 96, 107401 (2006).

[15] Nian-Hai Shen, Maria Massaouti, Mutlu Gokkavas, Jean-Michel Manceau, Ekmel Ozbay, Maria Kafesaki, Thomas Koschny, Stelios Tzortzakis, and Costas M. Soukoulis, "Optically Implemented Broadband Blueshift Switch in the Terahertz Regime,” Physical Review Letters 136, 037403 (2011). 